\title{
Evaluation of the efficacy of an attenuated live vaccine based on virulent porcine reproductive and respiratory syndrome virus 2 in young pigs
}

\author{
Seung-Chul Lee ${ }^{1,3}$, Yun-Hee Noh ${ }^{1}$, Sunhee Lee ${ }^{2}$, Hwan-Won Choi ${ }^{1}$, In-Joong Yoon ${ }^{1}$, \\ Shien-Young Kang ${ }^{3, *}$, Changhee Lee ${ }^{2, *}$ \\ ${ }^{1}$ Choong Ang Vaccine Laboratories Co., Ltd., Daejeon 34055, Korea \\ ${ }_{2}^{2}$ Animal Virology Laboratory, School of Life Sciences, BK21 Plus KNU Creative BioResearch Group, \\ Kyungpook National University, Daegu 41566, Korea \\ ${ }^{3}$ College of Veterinary Medicine, Chungbuk National University, Cheongju 28644, Korea
}

(Received: April 14, 2018; Revised: June 26, 2018; Accepted: July 12, 2018)

\begin{abstract}
The efficacy of the CA-2-MP120 vaccine, a cell culture-attenuated strain of virulent porcine reproductive and respiratory syndrome virus (PRRSV), was assessed in pigs. Despite the persistence of viremia in all vaccinated animals during the immunization period, the virus was not detected in vaccinated pigs following challenge. Furthermore, no pigs in the vaccinated group shed PRRSV nasally, orally or rectally throughout the experiment. Moreover, histopathological lung and lymph node lesions in the immunized group were much milder than those in the unimmunized and challenged group. These results indicated that CA-2-MP120 can provide effective protection against virulent wildtype PRRSV-2.
\end{abstract}

Keywords: attenuated vaccines, porcine reproductive and respiratory syndrome virus, treatment outcome, vaccination

Porcine reproductive and respiratory syndrome virus (PRRSV) is a globally notorious and widespread viral pathogen that causes major economic losses in the swine industry worldwide [5]. It is a small, enveloped, single-stranded, and positive-sense RNA virus that has been recently reclassified to the genus Porarterivirus within the family Arteriviridae of the order Nidovirales [1]. The most outstanding hallmark of PRRSV is its high genetic and antigenic diversity due to a swift mutation rate; accordingly, the current taxonomy divides the virus into two species: PRRSV-1 (European) and PRRSV-2 (North American) [1]. Furthermore, PRRSV continues to undergo extremely high degrees of genetic divergence not only among the two species but also within them, leading to significant phenotypic variations in the clinical presentation among field isolates [3, 12]. The genetic and clinical heterogeneity is considered a major obstacle for PRRSV vaccine development and ultimately making its control and elimination complicated.

Although the two species have been intermingled in Korean pig farms since 2006 [11], PRRSV-2 is more prevalent than PRRSV-1 in South Korea [7]. Furthermore, at least 4 different lineages of PRRSV-2 circulate predominantly in the country, and heterologous PRRSV-2 strains can simultaneously exist in a single farm $[2,7]$. In particular, virulent
PRRSV-2 lineage 1 [4] has been epizootic in South Korea since the early 2010s [2].

We have previously reported the virulence, immunogenicity, and genetic characteristics of the CA-2-MP120 virus strain that was obtained by sequentially passing the virulent Korean PRRSV-2 lineage 1 strain CA-2 through the African green monkey kidney epithelial cell line MARC-145 (100 passages) and cultured porcine alveolar macrophage (PAM) (additional 20 passages) cells [8]. In this present study, we assessed the efficacy of CA-2-MP120 as a genetically stable, live-attenuated vaccine strain against PRRSV-2 lineage 2 in host animals. This vaccine was efficacious against a challenge with the virulent parental CA-2-P20 strain and can serve as an adequate vaccine in herds.

The in vivo swine experiments described herein were performed at the ChoongAng Vaccine Laboratory Animal Facility under the guidelines established by its Institutional Animal Care and Use Committee (IACUC No. 150128-02). A total of 16 pigs (3 weeks old), cross breeds of the Great Yorkshire and Dutch Landrace, were obtained from a pig farm with a high health status and randomly divided into three groups. All animals were determined to be free of antibodies to PRRSV, swine influenza virus, and porcine circovirus 2. After one week of acclimatization, the pigs in

*Corresponding author

Tel: +82-53-950-7365, Fax: +82-53-955-5522

E-mails: changhee@knu.ac.kr (C Lee), sykang@cbu.ac.kr (SY Kang) 
Group $1(n=6)$ were immunized intramuscularly with $2 \mathrm{~mL}$ of CA-2-MP120 at a 50\% tissue culture infective dose per $\mathrm{mL}\left(\mathrm{TCID}_{50} / \mathrm{mL}\right)$ of $10^{6.0}$, whereas the challenge control (Group 2; $n=6$ ) and strictly negative control (Group 3; $n=4$ ) received only cell culture medium as a placebo. At 57 days post-immunization (dpi), each piglet (except the negative control pigs from Group 3) was challenged intramuscularly with $2 \mathrm{~mL}\left(10^{6.0} \mathrm{TCID}_{50} / \mathrm{mL}\right)$ of the CA-2-P20 strain [2, 8]. Clinical parameters, such as appetite, activity level, and respiratory signs were monitored daily throughout the study. The body weight and rectal temperature were measured at $0,3,6,8$, $14,21,28,35,54,61$, and $71 \mathrm{dpi}$, and blood as well as oral, nasal, and fecal swabs were collected at the same time points. All the animals were euthanized at 14 days post-challenge (dpc) for post-mortem examination as described previously [8].
To determine if the animals were viremic during vaccination and challenge, PRRSV levels in the sera of pigs were determined by virus titration using MARC-145 or PAMKNU cells as described previously [2, 8]. PRRSV-specific antibodies in serum samples collected from the infected and controls animals were determined using a commercial PRRSV antibody ELISA kit (HerdCheck PRRS X3; IDEXX Laboratories, USA) according to the manufacturer's instructions and a serum neutralization test on PAM-KNU cells using a homogeneous CA-2 strain as previously described $[2,8]$.

To evaluate the efficacy of the CA-2-MP120 vaccine in the natural host, pig vaccination and challenge experiments were conducted. None of the pigs in the vaccinated and strict negative control groups developed typical clinical signs throughout the immunization and challenge procedures (up to $71 \mathrm{dpi}$

A

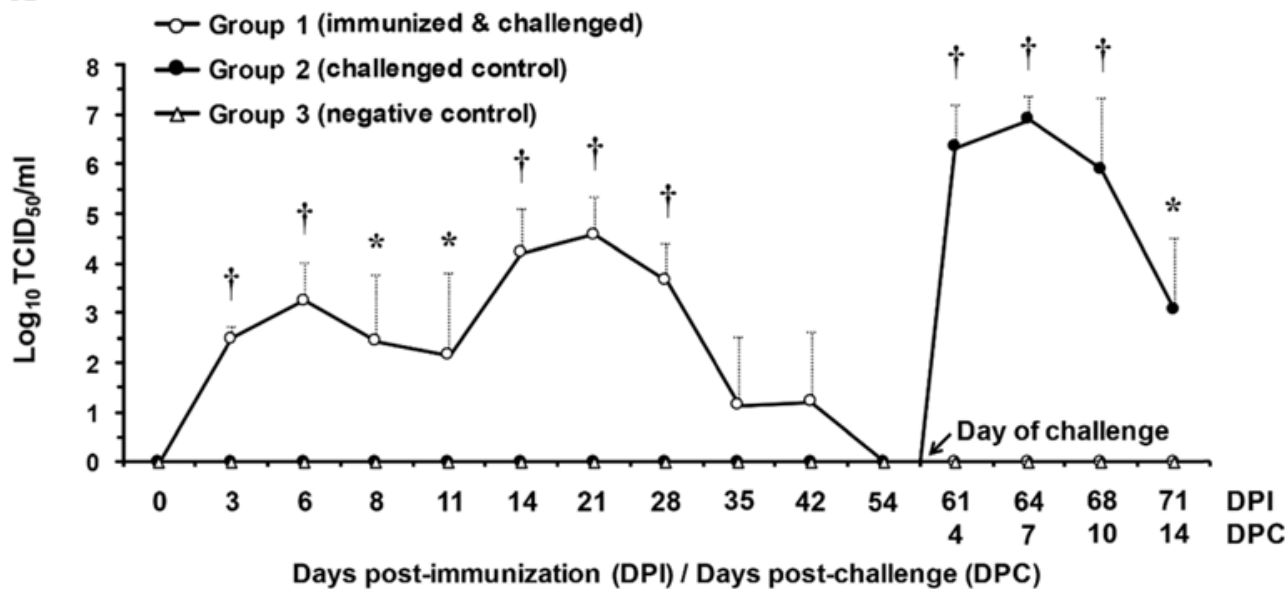

B

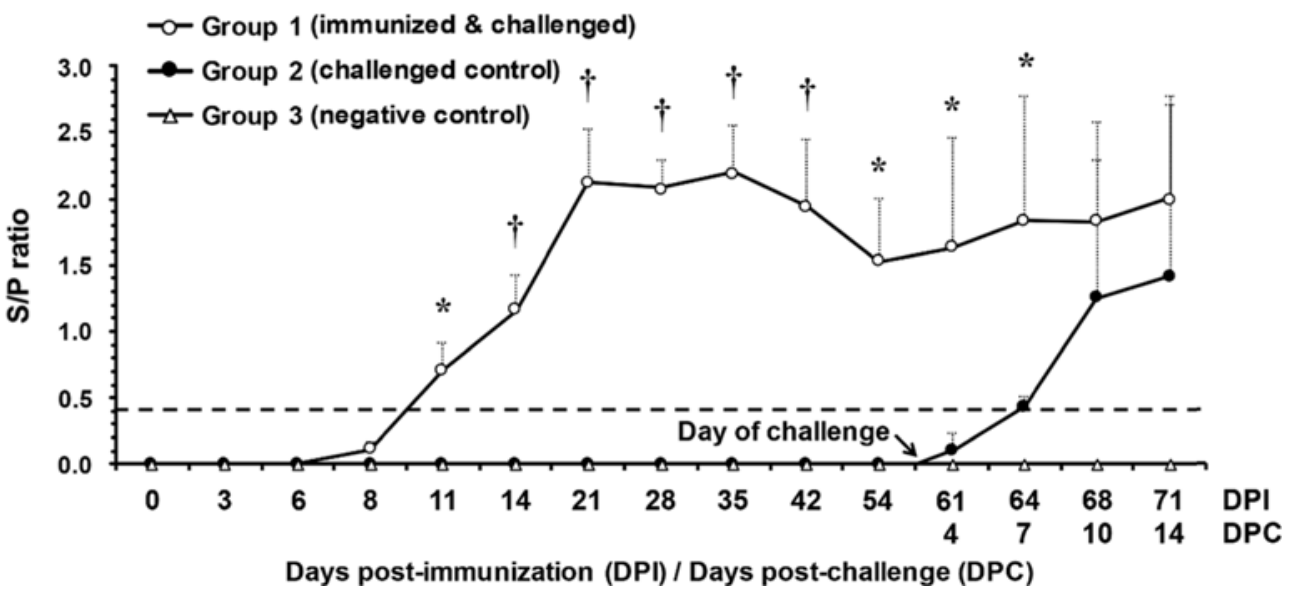

Fig. 1. Virus replication in vivo and seroconversion. (A) Mean levels of viremia of the immunized or unimmunized pigs after challenge. (B) Virus-specific antibody response of pigs, as measured by a commercial ELISA kit. Samples were considered positive for antibodies to PRRSV if the S/P ratio was equal to or greater than 0.4 . Error bars represent SD. $P$ values were calculated by comparing the immunized and unimmunized pigs using Student's $t$-test, and values of less than 0.05 were considered statistically significant. $* p<0.05 ; \stackrel{\dagger}{\dagger}<0.001$. 
or $14 \mathrm{dpc}$ ). However, following the virus challenge, some unvaccinated pigs (Group 2) exhibited mild clinical symptoms (e.g., dyspnea and anorexia) intermittently at 3-10 dpc, but eventually returned to a healthy state. Although pigs in the unvaccinated and challenged group had higher febrile responses and less weight gains during the post-challenge period compared with the immunized (Group 1) or negative control (Group 3) animals, there were no statistically significant differences in the mean rectal temperatures and average daily weight gains among the three groups throughout the experiment (data not shown).

Whereas all pigs in the vaccinated group developed viremia by $3 \mathrm{dpi}$, those in the unvaccinated controls (Groups 2 and 3) remained viremia-negative (Fig. 1A). Study of the viremia kinetics indicated that the viral replication levels of the immunized group had primarily declined at $8 \mathrm{dpi}$ but rebounded at $14 \mathrm{dpi}$ and then gradually decreased following the second peak time points at $28 \mathrm{dpi}$. Half of the pigs in Group 1 experienced viremia until 35 dpi, whereas the remaining animals were viremic until 42 dpi. Strikingly, PRRSV was undetectable in the sera of all vaccinated and challenged animals (6/6) throughout the post-challenge period, indicating that the challenge virus had been eliminated by the vaccine in the immunized group. Furthermore, none of the pigs in Group 1 shed the virus in nasal, oral, or fecal secretions during the entire immunization and challenge period. In contrast, all animals in the unvaccinated and challenged group developed viremia by $4 \mathrm{dpc}$, which continued until the termination of the experiment. Consistently, nasal, oral, and fecal virus shedding was recorded in piglets in Group 2 at different time points post-challenge.

Antibody detection by ELISA revealed that seroconversion had occurred by 11 dpi in all pigs in the group immunized with CA-2-MP120 and that the mean sample to positive ratio of the antibody test had reached its highest level at $21 \mathrm{dpi}$, followed by a decrease at $42 \mathrm{dpi}$ and ascending kinetics following challenge (Fig. 1B). Similarly, animals in the challenged control group had seroconverted by $7(4 / 6)$ or $10(2 / 6) \mathrm{dpc}$, and the antibody titers continued to rise thereafter. Serum samples from the strict negative control pigs (Group 3) remained seronegative to PRRSV throughout the trial, as determined by ELISA. Although a PRRSV-specific serum neutralizing (SN) antibody response was evident in all pigs in the vaccinated group at $14 \mathrm{dpc}$ with a mean titer of $1: 16$ ranging from $1: 8$ to $1: 36$, the vaccinated animals were negative for (until $4 \mathrm{dpc}$ ) or insignificantly low levels $(\leq 1: 4)$ of $\mathrm{SN}$ antibodies against the challenge virus at $7 \mathrm{dpc}$. These results suggested that the SN antibody response may play a limited role in protective immunity against PRRSV infection, particularly regarding the clearance of viremia in the present study. In addition, PRRSV-specific SN antibodies were found to be absent in all animals in the challenge (Group 2) and negative (Group 3) controls until the end of the experiment (data not shown).

As shown in Figure 2A, post-mortem examination revealed
A
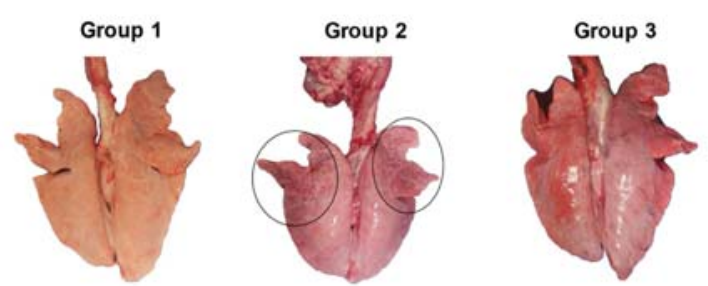

B

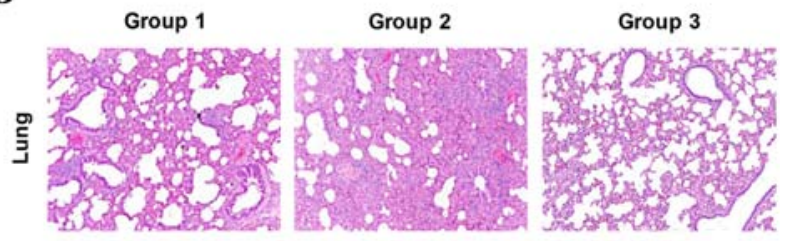

C

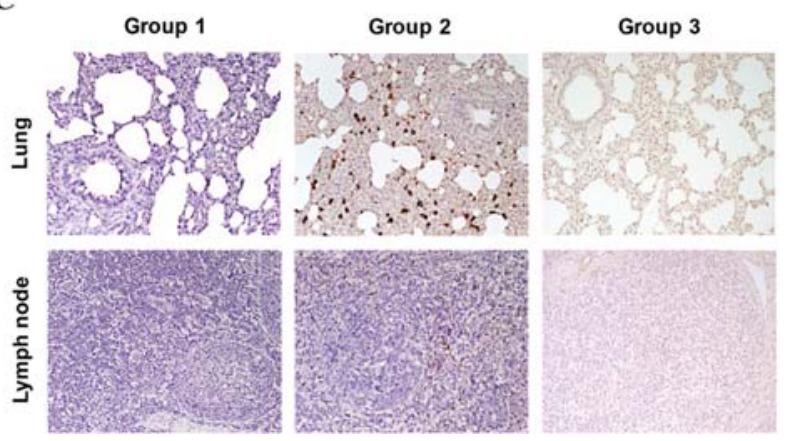

Fig. 2. Macroscopic and microscopic examinations of pigs from the three study groups. (A) Lungs from the representative pigs in each group were examined for gross lesions. Note that only piglets from the challenged control (Group 2) show interstitial pneumonia, and pathological lesions are circled. (B) Lung tissues from the pigs in each group were histopathologically examined by hematoxylin and eosin staining. The unimmunized and challenged pigs (Group 2) showed marked interstitial pneumonia with alveolar walls thickened by massive lymphocyte and macrophage infiltration, whereas the immunized and challenged pigs (Group 1) exhibited normal lung lesions comparable to those in the strict negative control pig (Group 3). (C) Detection of PRRSV antigen by immunohistochemical analysis of lung and lymph node (LN) tissue sections from pigs in each group. PRRSV antigen signals appeared brown and were detected in the lungs and LNs from pigs in the challenged control (Group 2). No PRRSV antigen was detected in both tissues of the pigs in Groups 1 and 3. 100× (B and C).

typical pulmonary lesions, including interstitial pneumonia with red-tan, rubbery, and firm areas in pigs in the challenged control (Group 2). In the vaccinated and challenged animals (Group 1), no pathological damage was found macroscopically, which was comparable to the results seen in the strict negative control at necropsy (Group 3). Upon microscopic assessment, the lungs from all pigs in the challenged control (Group 2) showed general characteristics of acute PRRSV infection, including thickening of the alveolar walls by the infiltration and accumulation of inflammatory cells in 


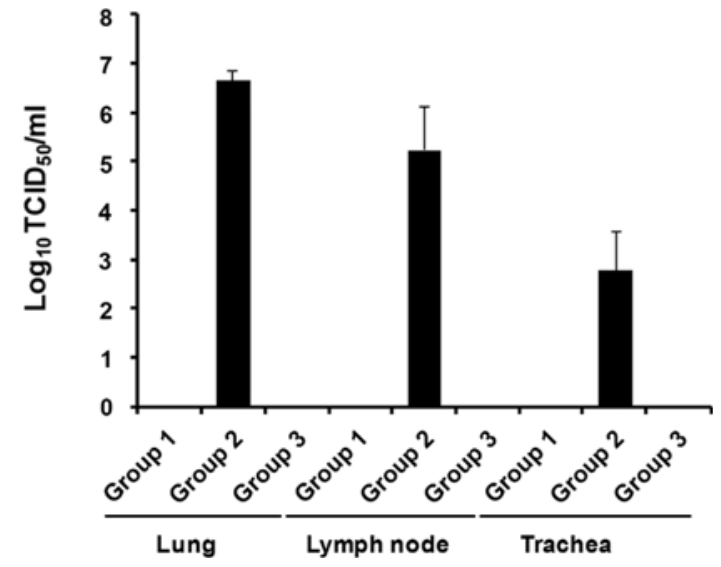

Fig. 3. Virus isolation in lungs, LN, and tracheas from pigs in the three study groups. No PRRSV was isolated from any of the tissue samples collected from the pigs in Groups 1 and 3.

the interstitial tissue. In contrast, the lungs of the immunized pigs in Group 1 displayed mild alveolar wall thickening without typical interstitial pneumonia (Fig. 2B). Animals in Groups 1 and 2 equally developed minimal or no remarkable lesions in the lymph nodes (LNs) or tracheas (data not shown). Immunohistochemical staining exhibited that the PRRSV antigen was predominant in the lungs and LNs of the challenged pigs in Group 2, whereas it was undetectable in the immunized pigs in Group 1 (Fig. 2C). Furthermore, virus isolation analysis showed that only pigs in the challenged control (Group 2) were positive for PRRSV in their lungs, LNs, and tracheas (Fig. 3). None of the pigs in Group 3 experienced any histopathological lesions or showed virus positivity in the lungs, LNs, and tracheas. Therefore, our results indicated that the attenuated CA-2-MP120 strain provided protection against virulent PRRSV infection in pigs.

Currently, several modified live-attenuated vaccines (MLVs) against PRRSV-1 or -2 have been successfully employed. All of the PRRSV vaccines are generally effective against homologous strains but show varying and sometimes incomplete effectiveness against heterologous strains [6, 9]. Moreover, acute PRRS-like disease and atypical PRRS characterized by abortion and high mortality in pregnant gilts were reported in MLV-vaccinated pigs. Notably, the virulent PRRSV-2 lineage 1 strain emerged and rapidly became prevalent in South Korea and plagued the domestic pork industry economically $[2,10]$. Therefore, it is imperative to develop safe and effective vaccines against the virulent field PRRSVs. The current study demonstrated that CA-2-MP120 is capable of providing effective protection against challenge with a virulent homologous virus. Although further challenge experiments will be needed to evaluate the protective efficacy of vaccines against a heterologous virus and to assess safety concerns with regard to reversion, our work provides valuable insights for pioneering the development of a next-generation MLV based on the attenuated CA-2-MP120 virus.

\section{Acknowledgments}

This research was supported by the Basic Science Research Program through the National Research Foundation of Korea (NRF) funded by the Ministry of Education (NRF-2015R1D1A1A09057406).

\section{References}

1. Adams MJ, Lefkowitz EJ, King AMQ, Harrach B, Harrison RL, Knowles NJ, Kropinski AM, Krupovic M, Kuhn JH, Mushegian AR, Nibert M, Sabanadzovic S, Sanfaçon H, Siddell SG, Simmonds P, Varsani A, Zerbini FM, Gorbalenya AE, Davison AJ. Changes to taxonomy and the International Code of Virus Classification and Nomenclature ratified by the International Committee on Taxonomy of Viruses (2017). Arch Virol 2017, 162, 25052538.

2. Choi HW, Nam E, Lee YJ, Noh YH, Lee SC, Yoon IJ, Kim HS, Kang SY, Choi YK, Lee C. Genomic analysis and pathogenic characteristics of Type 2 porcine reproductive and respiratory syndrome virus nsp2 deletion strains isolated in Korea. Vet Microbiol 2014, 170, 232-245.

3. Faaberg KS, Han J, Wang Y. Molecular dissection of porcine reproductive and respiratory virus putative nonstructural protein 2. Adv Exp Med Biol 2006, 581, 73-77.

4. Han J, Wang Y, Faaberg KS. Complete genome analysis of RFLP 184 isolates of porcine reproductive and respiratory syndrome virus. Virus Res 2006, 122, 175-182.

5. Holtkamp DJ, Kliebenstein JB, Neumann EJ, Zimmerman JJ, Rotto HF, Yoder TK, Wang C, Yeske PE, Mowrer CL, Haley CA. Assessment of the economic impact of porcine reproductive and respiratory syndrome virus on United States pork producers. J Swine Health Prod 2013, 21, 72-84.

6. Huang YW, Meng XJ. Novel strategies and approaches to develop the next generation of vaccines against porcine reproductive and respiratory syndrome virus (PRRSV). Virus Res 2010, 154, 141-149.

7. Lee JA, Lee NH, Lee JB, Park SY, Song CS, Choi IS, Lee SW. Genetic diversity of the Korean field strains of porcine reproductive and respiratory syndrome virus. Infect Genet Evol 2016, 40, 288-294.

8. Lee SC, Lee S, Yoo GW, Choi HW, Noh YH, Park CE, Shin JH, Yoon IJ, Kang SY, Lee C. Phenotypic and genotypic analyses of an attenuated porcine reproductive and respiratory syndrome virus strain after serial passages in cultured porcine alveolar macrophages. J Vet Sci 2018, 19, 358-367.

9. Leng X, Li Z, Xia M, He Y, Wu H. Evaluation of the efficacy of an attenuated live vaccine against highly pathogenic porcine reproductive and respiratory syndrome virus in young pigs. Clin Vaccine Immunol 2012, 19, 1199-1206.

10. Mengeling WL, Lager KM, Vorwald AC. Clinical consequences of exposing pregnant gilts to strains of porcine reproductive and respiratory syndrome (PRRS) virus isolated from field cases of "atypical" PRRS. Am J Vet Res 1998, 59, 1540-1544.

11. Nam E, Park CK, Kim SH, Joo YS, Yeo SG, Lee C. 
Complete genomic characterization of a European type 1 porcine reproductive and respiratory syndrome virus isolate in Korea. Arch Virol 2009, 154, 629-638.

12. Stadejek T, Oleksiewicz MB, Scherbakov AV, Timina AM, Krabbe JS, Chabros K, Potapchuk D. Definition of subtypes in the European genotype of porcine reproductive and respiratory syndrome virus: nucleocapsid characteristics and geographical distribution in Europe. Arch Virol 2008, 153, 1479-1488. 